\title{
Middle Aortic Syndrome with Superior Mesenteric and Bilateral Renal Artery Involvement: Unusual Type of Aortic Coarctation
}

\author{
JongSeon Park, MD \\ Division of Cardiology, Department of Internal Medicine, Veterans Health Service Medical Center, Seoul, Korea
}

\begin{abstract}
| ABSTRACT |
Middle aortic syndrome (MAS) is very uncommon vascular pathology characterized by a long segmental narrowing or obstruction of the abdominal and/or distal thoracic aorta, commonly involving with the visceral and renal arteries. This syndrome may be presented with various physical signs of coarctation of the aorta, including resistant hypertension, renal insufficiency and/or mesenteric ischemia. Here, we report a case of a 64-year-old man with severe hypertension. He was diagnosed with MAS associated with stenosis of visceral and renal vessels by use of computed tomography and magnetic resonance angiography.
\end{abstract}

(J Korean Soc Hypertens 2013;19(1):39-43)

Key Words: Aortic coarctation; Abdominal aorta; Hypertension

\section{Introduction}

Aortic coarctation is a common congenital heart defect and occupies $5 \%$ to $10 \%$ of congenital heart disease. ${ }^{1)}$ In general, typical coarctation is usually located at the attachment of the ductus arteriosus to the thoracic aorta near the left subclavian artery. However, coarctation of the abdominal and/or distal thoracic aorta, referred to as a typical coarctation or middle aortic syndrome (MAS), is particularly rare in elderly patients. It accounts for less than $2 \%$ of all coarctacion cases. ${ }^{2-5)}$ Etiology of MAS may be congenital or acquired. ${ }^{1-5)}$ In this article, we de-

Received: 2013.2.15, Revised: 2013.3.26, Accepted: 2013.3.26

Correspondence to: JongSeon Park, MD

Address: Division of Cardiology, Department of Internal Medicine, Veterans Health Service Medical Center, 53 Jinhwangdo-ro 61-gil, Gangdong-gu, Seoul 134-791, Korea

Tel: +82-2-2225-1304, Fax: +82-2-2225-1491

E-mail: neo00100@daum.net scribe a 64-year-old man presenting with severe hypertension, diagnosed with MAS associated with superior mesenteric and bilateral renal artery stenosis.

\section{Case Report}

A 64-year-old gentleman was admitted with symptoms of headache, leg weakness with exertion, and dyspnea. His previous medical history included uncontrolled hypertension against several medications and cerebellar infarction. At admission, his mentality was alert and he had no focal neurological symptoms or signs. Physical examination revealed a discrepancy of blood pressure (BP) between arms and legs. The brachial BP was found 190/95 mm $\mathrm{Hg}$ in right arm, and 196/97 mm Hg in left arm. The thigh BP was 150/80 mm Hg. No abdominal bruit was detected. A 12-lead electrocardiogram revealed 

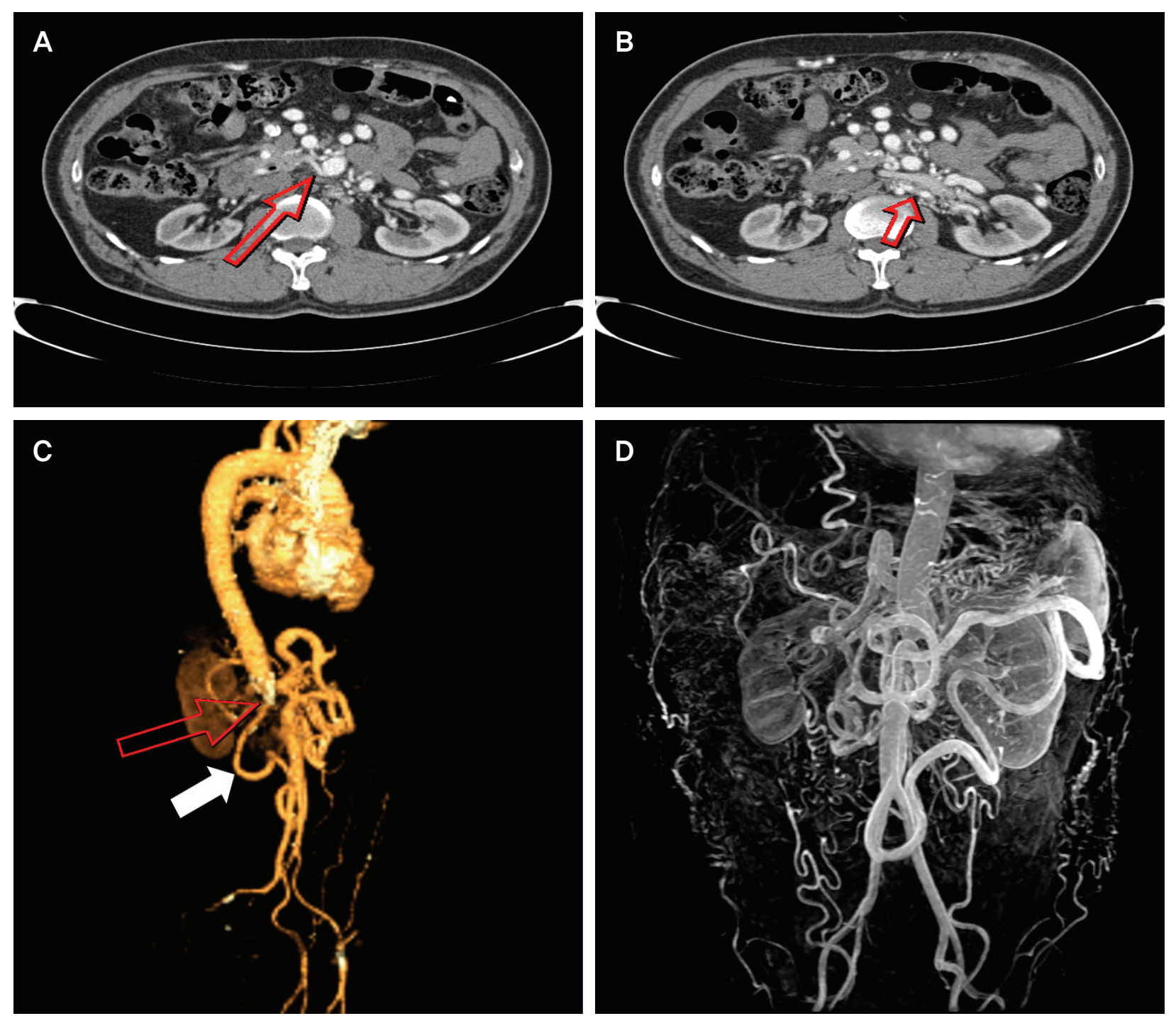

Fig. 1. Imaging of computed tomography (CT) and magnetic resonance angiography (MRA). (A) Origin of right renal artery (arrow) was not found around aorta, supplied by branch vessels arose from distal superior mesenteric artery and left renal artery. (B) Left renal artery (arrow) originating from hypoplastic abdominal aorta was severely calcified and stenosed. (C) Abrupt luminal obstruction of abdominal aorta just below the celiac trunk (Upper arrow). Superior mesenteric artery is supplied by the arc of Riolan (Lower arrow). Both iliac arteries are normal. (D) MRA showing similar but more detailed vasculature as compared with CT.

poor $\mathrm{R}$ wave progression across the precordium with left ventricular hypertrophy $(\mathrm{LVH})$ and chest $\mathrm{x}$-ray was normal. Computed tomography (CT) scan of the brain showed no acute lesion except multiple lacunar infarction and chronic ischemic change of the cerebellum. Transthoracic echocardiography (TTE) showed concentric LVH and mildly decreased LV systolic function without regional wall motion abnormality. No associated cardiac anomaly was seen on TTE. Blood chemistries including renal function test were within normal range, with a blood urea nitrogen level of $23 \mathrm{mg} / \mathrm{dL}$ and a creatinine level of $1.0 \mathrm{mg} / \mathrm{dL}$. Inflammatory markers, such as C-reactive protein, erythrocyte sedimentation rate, and white blood cell count, were normal. Abdominal ultra- 
sonography revealed multiple vasculatures around pancreas with focal nonvisualization of abdominal aorta. Contrast-enhanced CT scan of thoracoabdominal aorta demonstrated abrupt luminal obstruction of abdominal aorta just below the celiac trunk (Fig. 1C). Left renal artery originated from hypoplastic abdominal aorta was severely calcified and stenosed (Fig. 1B). On the other hand, origin of right renal artery was not found around aorta, supplied by branch vessels arose from distal superior mesenteric artery (SMA) and left renal artery (Fig. 1A). Celiac trunk and inferior mesenteric artery were spared, however origin of SMA was obliterated, supplied by the arc of Riolan and the arc of Buhler. The hypoplastic abdominal aorta was terminated after giving off the left renal artery. Bilateral iliac arteries were normal. We also performed contrast-enhanced magnetic resonance angiography (MRA) to obtain additional information including aortic branches and MRA showed similar but more detailed vasculature (Fig. 1D). Although he has taken four antihypertensive agents (angiotensin receptor blocker, calcium channel blocker, beta-blocker, and diuretics), the brachial BP was still high. We decided to conduct a surgery, but he refused for the personal reason. Finally he had six kinds of antihypertensive drugs with additional aldosterone antagonist and direct vasodilating agent and was discharged. The mean brachial BP during follow-up was 159/85 mm $\mathrm{Hg}$ and his symptoms such as headache and dyspnea showed more improvement. Two-year follow-up course since discharge was uneventful.

\section{Discussion}

Coarctation of the aorta is defined as segmental luminal narrowing of the aorta that produces hemodynamically significant blood flow obstruction. ${ }^{2)}$ Since the term "coarctation" is used exclusively for the designation of congenital vascular abnormality in the literature, we think
MAS has more comprehensive and broader meaning. MAS was first introduced by Sen et al. ${ }^{3)}$ who reported 16 cases associated with blocks in the middle segment (between the arch and the terminal bifurcation) of the aorta in 1963. MAS is a clinical condition which involves abdominal and/or distal descending thoracic aorta with its major branches including renal and mesenteric arteries, and is extremely rare. ${ }^{1,2,4)}$ It is slightly more common among females and is usually diagnosed between the first and third decades with a second peak in the fourth to fifth decsde. ${ }^{2,4)}$ The etiology of this condition is not clear, which may be congenital or acquired. Proposed mechanisms include developmental defect, response to infection, and inflammation. It may be associated with Takayasu's or temporal arteritis, neurofibromatosis, fibromuscular dysplasia, Williams syndrome, retroperitoneal fibrosis, maternal viral infection such as rubella, syphilis, tuberculosis, and radiation therapy or developmental anomaly. ${ }^{1-5)}$ In the present case, pathology seems to be more likely a combination of congenital hypoplasia with atherosclerotic change because the patient was diagnosed at 6th decade and any obvious inflammatory process was not seen in the blood sample and in imaging studies such as CT and MRA. The clinical manifestation of MAS is mainly severe hypertension in the upper part of the body proximal to the stenotic lesion. The hypertension which is most commonly presented in suprarenal or interrenal aortic coarctation on anatomic classification, is most likely to be due to global renal hypoperfusion and activation of rennin-angiotensin system. ${ }^{1,4,5)}$ Uncontrolled hypertension can result in $\mathrm{LVH}$, stroke, coronary artery disease, and congestive heart failure. In addition, patients may have claudication of the lower limb, headache, fatigue, epistaxis, and so on.

Albeit aortography has been a diagnostic tool of choice historically, CT and MRA are increasingly used as alternative tests. These imaging modalities cannot only dem- 
onstrate the location and extent of the aortic disease but also detect associated abnormalities of visceral and renal arteries. However, there are some limitations which include the lack of dynamic representation of flow abnormalities and difficulty in evaluation of heavily calcified arteries in CT, and less sensitivity in evaluation of smaller branches than major branches of aorta in MR. ${ }^{6,7)}$

The prognosis of MAS is generally poor in untreated cases, depending on the anatomic location to the renal arteries, the functional degree of involved arterial branches, and severity of hypertension. Actually, most of untreated patients succumbing before the age of $40 .{ }^{5)}$ A surgical repair such as aorto-aortic bypasss grafting or patch aortoplasty with visceral reconstruction, is the primary treatment of MAS associated with renovascular hypertension and visceral artery stenosis. ${ }^{4,5)}$ After surgery, their hypertension symptoms were either improved or cured in more than $70 \%$ of patients. ${ }^{5)}$

In the last three decades, endovascular treatment has become more widespread, using either balloon angioplasty or primary stent implantation. In adults, a stent placement after balloon angioplasty reduced the complications associated with angioplasty, such as recoarctation, dissection, and aneurysm formation. However, catheter interventions have been used only in selected cases with discrete lesions not involved with the renal and mesenteric arteries. ${ }^{5)}$ In Korea, seven cases of MAS in adults, including 4 cases without stenosis of major branches of aorta, were reported, among which renal artery involvement were in 3 , visceral artery involvement in 2 , and five patients were successfully treated with surgery. ${ }^{8-10)}$ In this case, simultaneous revascularization procedure for aorta and bilateral renal arteries is required to control his high BP. However, endovascular therapy was thought to be inappropriate due to aortic occlusion with complex collateral vessels and stenosis of both renal arteries of which one is totally obstructive and the other is severely calcified. Considering these factors, open surgical reconstruction was believed to be more suitable. However, surgery itself was quite challenging in this patient, because he had many risk factors such as relatively older age, reduced cardiac function and previous history of stroke, compared with previous cases. In addition, according to other article, in-hospital mortality following renovascular surgery in atherosclerotic disease has been reported up to $10 \%$, with the risk being increased in patients with diffuse atherosclerosis and heart failure. ${ }^{11)}$ The mortality rate seems to be similar renal bypass graft alone or combined with abdominal aortic repair or bypass. ${ }^{4,5)}$

Unfortunately, the patient did not consent to have surgery. Therefore, we decided to treat him with medication. Currently the patient maintains 159/85 mm $\mathrm{Hg}$ of BP. If we put the fact that the patient did not underwent revascularization into consideration, the BP appears to be adequately controlled to maintain renal blood flow and glomerular filtration rate in the context of bilateral renal stenosis. ${ }^{12)}$

In spite of the fact that MAS is a rare disease, there are approximately 200 cases related to MAS worldwide. ${ }^{5)}$ In Korea, however, there are only four reported cases having involvement of mesenteric or renal arteries, all of which, in our opinion, did not provide a detailed description of complex anatomies with CT and MRA findings. Therefore, we would like to describe the case of a 64-year-old man diagnosed with MAS involving SMA and both renal arteries by use of CT and MRA. Because of the risk factors for surgery itself and the patient's refusal to receive surgery, we had to choose medical treatment. This case showed that although the primary treatment option for patients having MAS with resistant renovascular hypertension is to have surgery, the medical treatment alone may be the second best strategy in con- 
trolling the patient's BP level and improve the severity of the patient's condition. As previously described, these patients usually die due to cardiac or cerebrovascular complications before the age of 40 , if left untreated. But our patient has had comparatively stable condition until now, despite his old age. Constant clinical monitoring should be required for further outcome evaluation.

\section{Conflict of interest}

No potential conflict of interest relevant to this article was reported.

\section{References}

1. Celik T, Kursaklioglu H, Iyisoy A, Turhan H, Amasyali B, Kocaoglu M, et al. Hypoplasia of the descending thoracic and abdominal aorta: a case report and review of literature. J Thorac Imaging. 2006;21:296-9.

2. Terramani TT, Salim A, Hood DB, Rowe VL, Weaver FA. Hypoplasia of the descending thoracic and abdominal aorta: a report of two cases and review of the literature. J Vasc Surg. 2002;36:844-8.

3. Sen PK, Kinare SG, Engineer SD, Parulkar GB. The middle aortic syndrome. Br Heart J. 1963;25:610-8.

4. Cohen JR, Birnbaum E. Coarctation of the abdominal aorta. J Vasc Surg. 1988;8:160-4.
5. Delis KT, Gloviczki P. Middle aortic syndrome: from presentation to contemporary open surgical and endovascular treatment. Perspect Vasc Surg Endovasc Ther. 2005;17:187-203.

6. Ilica AT, Bilici A, Ilhan A, Kara M, Gur S. Thoracoabdominal aorta coarctation with bilateral renal artery involvement: diagnosis with multidetector $\mathrm{CT}$ angiography (MDCTA). Int $\mathrm{J}$ Cardiovasc Imaging. 2007;23:645-8.

7. Tosun O, Sanlidilek U, Cetin H, Ozdemir O, Kurt A, Sakarya $\mathrm{ME}$, et al. Unusual congenital aortic anomaly with rare common celiamesenteric trunk variation: MR angiography and digital substraction angiography findings. Cardiovasc Intervent Radiol. 2007;30:1061-4.

8. Kim SJ, Ha J. Middle aortic syndrome: report of 4 cases. Korean J Vasc Endovasc Surg. 1995;11:230-7.

9. Kim DK, Kim YJ, Ryu JS, Eom WS, Cho JH, Jeong YT. Middle aortic syndrome diagnosed at 51 years of age. Korean J Med. 2004;66:293-7.

10. Kim JA, Kim DB, Jang SW, Kwon BJ, Cho EJ, Song JH, et al. Hypertensive heart failure with severe arteriosclerotic stenosis of the descending aorta. Korean Circ J. 2007;37:590-3.

11. Modrall JG, Rosero EB, Smith ST, Arko FR 3rd, Valentine RJ, Clagett GP, et al. Operative mortality for renal artery bypass in the United States: results from the National Inpatient Sample. J Vasc Surg. 2008;48:317-322.

12. Textor SC, Novick AC, Tarazi RC, Klimas V, Vidt DG, Pohl M. Critical perfusion pressure for renal function in patients with bilateral atherosclerotic renal vascular disease. Ann Intern Med. 1985;102:308-14. 